\title{
Ions Interacting in Solution: Moving from Intrinsic to Collective Properties
}

\author{
Timothy T. Duignan ${ }^{\mathrm{a}, *}$, Marcel D. Baer ${ }^{\mathrm{a}}$, Christopher J. Mundy ${ }^{\mathrm{a}}$
}

${ }^{a}$ Chemical and Materials Sciences Division, Pacific Northwest National Laboratory, P.O. Box 999, Richland, Washington 99352, USA

\begin{abstract}
A crucial determinant of Hofmeister effects is the direct interaction of ions in solution with the charged groups on the surface of larger particles. Understanding ion-ion interactions in solution is therefore a necessary first step to explaining Hofmeister effects. Here, we advocate an approach to modeling these types of properties where state of the art $A b$ Initio Molecular Dynamics (AIMD) simulation of ions in solution is used to establish benchmark values for the intrinsic properties of ions in solution such as solvation structures and ion-ion Potentials of Mean Force (PMFs). This information can then be combined with or used to parametrize and improve reduced models, which use approximations such as the continuum solvent model.(CSM) These reduced models can then be used to calculate collective and concentration dependent properties of electrolyte solution and so make accurate predictions about complex systems of relevance for direct applications. We provide an example of this approach using AIMD calculations of the sodium chloride dimer to calculate osmotic coefficients of all 20 alkali halide electrolytes.
\end{abstract}

Keywords: Hofmeister Effects, osmotic coefficients, activity coefficients, ion-ion interactions, Potentials of Mean Force.

\section{Introduction}

Modeling the interaction of ions in solution is one of the most fundamental and longstanding problems of physical chemistry, but unfortunately, there is still much that we do not understand about this problem. In particular, modeling the ion specificity of these interactions often requires resorting to fitted parameters for each cation-anion pair, severely limiting the usefulness of our models. Solving this problem is a crucial hurdle to cross before predictive and quantitative models of Hofmeister effects can be built. In this article, we review the emerging state-of - the-art in both the use and construction of accurate molecular-based models of ion-ion interaction to inform our understanding of ion-pairing.

In the past five years, there has been a resurgence of theoretical interest in constructing reduced or coarsegrained models to explain ion or solute specific effects. 1 6. Although the details of the construction of the models is distinct, a common approach is to make use of fully molecular-based frameworks where explicit details can be exported as input into the reduced models. These explicit molecular level details we will define as the intrinsic properties of ions in solution. They are essentially properties of the isolated ion or ion-pair in solution. They can come in the form of ionic radii and polarizability, solvation free energies, coordination numbers, Potentials of Mean Force (PMFs) at infinite dilution, or a general classification of

\footnotetext{
${ }^{*}$ Corresponding author

Email addresses: Timothy.Duignan@pnnl.gov (Timothy T. Duignan), Marcel.Baer@pnnl.gov (Marcel D. Baer), Chris.Mundy@pnnl.gov (Christopher J. Mundy)
}

ions as "kosmotropes" or "chaotropes." This intrinsic information is generally of a local or short-range (SR) character due to system size limitations of molecular level descriptions. Once the intrinsic properties have been determined, they can be combined with or used to inform and parametrize reduced models, which can then be used to predict concentration dependent and collective phenomena ranging from osmotic and activity coefficients [7] to surface forces and colloidal stability. 4]

An important aspect of this article is to review and articulate the challenges of connecting the fully molecular to the reduced continuum theoretical frameworks. In particular we focus on the ion-ion interactions in solution and show how a detailed and rigorous molecular understanding can be utilized to improve reduced models and develop our understanding of the collective behavior of electrolyte solutions. We believe this approach should eventually move us significantly closer to the goal of developing a reduced quantitative model of the collective properties of electrolyte solutions.

The role of experiment is also crucially important. Specifically, the thermodynamic properties of electrolyte solutions, such as osmotic coefficients and solvation free-energies, have long been established. The connection of the collective properties of electrolytes to the experimentally determined SR molecular structure is beginning to be established through the combination of extended X-ray absorption fine structure (EXAFS) in conjunction with $\mathrm{X}$-ray diffraction (XRD). [8] These exquisite and sensitive experiments are now able to resolve details accurately enough that they can be used to test the quality of different interaction potentials. 
Herein, we outline an approach for connecting the molecular to the continuum frameworks that highlights how to use information from a variety of modeling approaches to build a better understanding of ion-specific interactions. In particular, we emphasize the use of $a b$ initio models based on quantum density functional theory (DFT) that give a complete, rigorous, and accurate description of the intrinsic properties of ions in solution. We demonstrate an example of this approach where we use information from Ab Initio Molecular Dynamics (AIMD) DFT calculations of the short-range ion-ion interaction in solution to inform continuum solvent models (CSMs) of ion-ion interactions that can be used to calculate experimental osmotic coefficients. We show impressive agreement with experimentally determined osmotic coefficients of alkali halide salts.

\section{Applications of ion-ion interactions}

The interaction of ions in solutions, particularly water, is of central importance in a wide range of applications. This is firstly because the direct interaction of simple ions in solution is important in determining the activities of ions, which determine chemical equilibria of ionic species, relevant for numerous applications. Two examples are carbonate ions [9] and radioactive ions in solution. [10] These direct ion interactions also determine osmotic pressures of salt solutions, which are central in desalination applications. 11

But the importance of these interactions goes far beyond the cases where they are directly relevant. This is because without an understanding of the binding of simple ions in water, we have no hope of fully understanding the interaction of charged groups in more complex situations. These charged groups exist on many proteins and on many mineral surfaces such as silica. The interactions of these charged groups with each other and with ions in solution are closely related to simple ion-ion interactions in solution. These interactions are crucial because there is a consensus that the direct interaction of ions with macromolecules plays a dominant role in determining the Hofmeister Series. 12 18] This is clear from the fact that these ionic interactions will determine the effective charge on interfaces and proteins when they are immersed in electrolyte. These surface charges in turn determine surface forces and potentials, as well as solubilities and protein conformation. These properties exemplify the classic Hofmeister effects. The calculation of ion-ion interactions in any situation is, therefore, a central prerequisite to predictive quantitative models of Hofmeister effects. Ref. 18 provides an excellent example of how direct ionic interactions can be used to reproduce the classic Hofmeister series. Understanding Hofmeister effects is, in turn, necessary to model crosscutting applications of the physical chemistry of solutions to biology, energy storage, and synthesis, for example, materials assembly processes and synthesis, particularly at the nanoscale. 19] Predicting the interactions of charged groups on larger molecules with each other is also essential for understanding many systems, for example, salt-bridge formation is a central mechanism in protein folding. 20]

A very closely related problem is the binding of ions to highly polar molecules, for example the binding of cations to the amide carbonyl in $N$-methylacetamide (NMA), which was recently investigated in Ref. 21. Both experiment and molecular simulation came into play in this study. In particular, recent infrared (IR) measurements of the amide I shifts of sodium versus calcium salts suggest a picture where the calcium cation is a strongly perturbing influence whereas the sodium cation is not. Direct comparison of the PMFs from classical and DFT-based interaction potentials showed significant quantitative differences, and DFT more satisfactorily reproduced IR measurements. This study highlights many of the objectives that we have set out to review in this opinion. It showcases the use of molecular theory and the direct calculation of experimental probes such as IR to further a structurefunction relationship based on intrinsic properties of a model peptide with a variety of ions.

The self-consistent coupling between the dielectric response of a surface and the electrolyte is crucial to determining how interfaces alter the pairing and partitioning of free ions in their vicinity. Studies that examine the effects of an (air-water) interface on ion-pairing are now being undertaken. 22] However, again, these important studies have been initiated with rigid point charge models, and the explicit coupling between the inhomogeneity of the interface and the form of the molecular interaction is not present. This situation has been remedied in the form of a dielectric boundary condition that accounts for the exact electrostatic coupling between a molecular liquid and a dielectric boundary. 23. It should be possible to extend this kind of boundary conditions to DFT studies to account not only for electrostatic response but the electrodynamic response, which will yield modified dispersion forces. The results of these studies will treat both dispersion and electrostatics self-consistently allowing for a molecular view of the formation of a double layer. This will improve on the commonly used mean-field Guoy-Chapman models of the double layer.

\section{Intrinsic to Collective}

The question then is how best to model the aforementioned important processes. To strive toward a complete quantitative understanding of collective properties of solutions, it is essential to build models that first reproduce the known intrinsic properties of the ion.

This means firstly reproducing the structural properties of the ion in water, i.e., the reproduction of EXAFS and XRD spectra are crucial tests of models. 8, 24 Secondly, it means reproducing thermodynamic properties of electrolyte solution at infinite dilution. The most important example of this is the solvation energies, but solvation entropies, partial molar volumes and other properties are 
also important.25 Ideally reproducing the correct structural information will directly lead to reproducing the correct thermodynamic properties of ions.

This approach is not a particularly novel idea, it has been applied by several researchers independently, and is a common feature of many of the most successful models of ions in solution. For instance, AIMD simulations have been compared in detail with EXAFS measurements. 24, 26 30 The parameters for Classical Molecular Dynamics (CMD) simulations have been fitted to reproduce ionoxygen radial distributions from XRD and solvation energies and entropies 31, 32, as have CSMs.33. This approach is not, however, as widely applied as it should be. Too often models are built and parametrized to explain systems with complex collective behaviors, without first testing the model by applying it to the most fundamental and simplest possible cases, such as the intrinsic properties of ions in solution.

\subsection{Intrinsic solvation energy}

The solvation free energy of ions is a particularly important example of an intrinsic property of an ion. This is clear from the fact that free energies of interaction of ions in solution can essentially be thought of as a change in the solvation energy of the pair of ions as they approach each other. 34]

The challenges of correctly modeling the solvation freeenergies of an ion in solution brings up the importance of understanding the role of interfaces in the partitioning of the free-energy of ion solvation. Schematically, one can envision the free-energy of solvation of an ion as comprised of two distinct pieces. The first is determined by the direct interaction of the ion with the surrounding solvent as well as the energy of forming a cavity for the ion to occupy. This is commonly referred to as the intrinsic solvation energy. The subtle and presently controversial part of free-energy of solvation comes from the contribution of the ion crossing the potential drop caused by the charge distribution at the air-water interface. This is commonly referred to as the surface potential of the air-water interface, and unfortunately it may contribute significantly to real solvation energies of ions in solution. Moreover, the size and even sign of this effect is still the subject of significant debate in the literature. 35] As a result, it is not possible to say with confidence that any model is correctly reproducing the intrinsic solvation energies of a single ion in solution. This significantly limits the confidence we can have in any model of charges in solution. Resolving this somewhat esoteric debate is a crucial prerequisite to be able to model any system where charged groups interact in solution.

Hünenberger argues that $-1100 \mathrm{kJmol}^{-1}$ is the correct value for the intrinsic solvation energy of a hydrogen ion, where the term intrinsic indicates that the contribution from the surface potential of the air-water interface is not included. This is based on a number of independent lines of evidence, which converge on this value. We believe these are the gold standard values for this quantity. A contrary view point 35 is that the Quasi-Chemical Theory (QCT) as applied in Ref. 36, 37, gives the correct intrinsic solvation energy. This requires that air-water interface has a surface potential of $-0.5 \mathrm{~V}$. These values are close to the ones provided by Marcus 38 and Schmid. 39] We believe that Ref. 34 provides a compelling critique of this QCT estimate of the solvation energy. It shows that the monomer thermodynamic cycle, which QCT uses, accumulates error as the number of water molecules increases. In contrast, a cluster cycle is more accurate and converges with the number of water molecules. Additionally the value of the air-water interface can be approximated using DFT interaction potentials. [40] These calculations indicate that the surface potential due to the air-water interface is significantly smaller than the $-0.5 \mathrm{~V}$ predicted with CMD molecular structures.

An important example of the direct difficulties this problem causes is shown in Ref. 31. Where Lennard-Jones parameters are adjusted to reproduce solvation energies, but because the intrinsic solvation energies are not accurately known, the parameters have to be fitted to reproduce solvation energies of cation-anion pairs, significantly increasing the uncertainty in the model. In addition, Ref. 31 shows that it is not possible to simultaneously reproduce solvation energies, entropies and radial distribution function peak positions with simple CMD models for the anions, perhaps highlighting a fundamental limitation of this approach. A possible solution is to build models that include the effect of the surface potential and test that they reproduce experimental real solvation energies of ions. These values are known with much more certainty. 25]

Currently, calculations of ionic solvation energies with AIMD simulation are extremely rare. Ref. 41 is the only example we are aware of. This is therefore an important area of future work as it is an important step to test and confirm that the models are correct beyond just reproducing structural properties.

\subsection{Ion-Surface Interaction}

Another crucial but slightly more sophisticated intrinsic property of ions in solution is the PMF of an ion as it approaches an air-water interface. This has been the topic of intense theoretical interest in recent years. AIMD calculations of the PMFs of ions at the air-water interface have been performed. 42 46, The resulting iodide potentials are consistent with theoretical studies based on reduced continuum description that can reproduce the experimentally observed ion specific surface tension increments for monovalent salts. 3, 47] In particular, by basing the model on an accurate calculation of solvation energies Ref. 33 was able to reproduce the surface tension increments with one consistent framework. This model also showed promise when applied to hydronium and hydroxide, reproducing the surface tensions increments of these salts accurately. [48] 
These theoretical models assume a near zero surface potential of the air-water interface. Their consistency with the AIMD calculations therefore supports the small value for the surface potential contribution we advocated for above. The CMD water models of Ref. 31 mentioned above has also been used to calculate ion PMFs with the interface that are relatively consistent with the AIMD and CSM calculations. In particular, the well-depth matches the AIMD and CSM calculations. 49 However this does depend quite significantly on which fundamental properties the Lennard-Jones properties are adjusted to reproduce. This model also qualitatively reproduces the correct ion-specific surface tensions increments. 31.

\subsection{Ion-Pairing}

Once a model that reproduces the most basic intrinsic properties of an ion has been developed, the next step is to generalize the model to pairs of ions. Essentially, this amounts to treating an isolated pair of ions alone under bulk aqueous solvation. The essential property of interest here is the PMF between the two ions. It should be possible to use these infinite dilution or intrinsic PMFs to provide an accurate description of the collective properties of electrolyte solutions. Clearly, at high concentrations the intrinsic PMF is no longer a good descriptor and manybody correlations will become important to describe the collective properties of electrolyte solutions. Ref. 2] shows this transition nicely, by using force matching to extract an effective ion-ion two body interaction and showing how it changes with concentration. It seems to be the case that somewhere above $1 \mathrm{M}$ the simple infinite dilution PMF begins to break down.

The most common and oldest approach to modeling ion-ion interactions in solution is to use the continuum solvent approximation starting with the Debye Hückel (DH) model for the mean field interaction of ideal charges in solution. Studies have augmented this mean field potential using additional terms with parameters fitted to reproduce experiment. Some examples are hard sphere, 50 Gurney, [51] and dispersion potentials. [52] A key problem with these approaches is they do not build on an accurate model of more basic intrinsic properties of an ion in solution as discussed above. More specifically they do not make any attempt to reproduce the solvation energy of the ion. In contrast, Ref. 53] calculates ion-ion interactions by generalizing a successful CSM of ionic solvation energies, 33 entropies and partial molar volumes. [54]

Although, the CSM of Ref. 53 shows a strong over attraction of ions, it does reproduce ion-specific trends nearly quantitatively when the potentials are damped, with two fitted parameters. This over attraction is a common problem with CSMs of ion-ion interactions 20 and, to a lesser extent, is also a problem with classical simulation. The Electronic Continuum Correction with Rescaling (ECCR) 55, 56 is aimed at fixing this problem. The calculation of ion-ion interactions in solution has been a focus of CMD for quite some time. 57. The ion-ion interactions in solution calculated with CMD do show a strong dependence on the parameters used for the model. [30, 58] Some success, however, has been observed using CMD to reproduce the ion-ion interaction trends qualitatively. [59] However, to quantitatively reproduce experimental activities, it is necessary to adjust salt specific parameters, 60. 61 which is obviously a serious problem that requires a solution beyond adjusting parameters for every possible ion-ion interaction.

As outlined above, AIMD simulations with DFT can in principle provide much more accurate potentials with almost no empirical parametrization. Although it is well known that DFT has somewhat serious deficiencies, recent studies have made great progress in showing that DFT plus empirical corrections to dispersion can provide unprecedented accuracy into the prediction of the intrinsic solvation structure of a variety of anions and cations. 24, 26] Thus, there is an emerging consensus that DFT can provide a superior description of local solvation structure over classical empirical interaction potentials and can even provide benchmarks to determine what classical water model is best. [40] Moreover, a recent DFT study of the ionpairing of calcium chloride 30 provided a self-consistent treatment of the intrinsic properties of the calcium cation and chloride anion both as individual ions and as a pair of ions. The results agreed with EXAFS and XRD studies [8] as well as with refitted classical empirical potentials that were adjusted to reproduce collective properties of calcium chloride. 55, 62

Despite these successes however, it is unlikely that the use of a single framework such as either molecular dynamics or a continuum based theory will yield the general and quantitative picture that is necessary to build an understanding of Hofmeister effects. For example, the calculation of ion-pair PMFs out to large separation with AIMD is difficult and requires extensive computational resources limiting their application to only a few cases so far. 30, 63, 64 Practical simulation issues that are based in sampling, finite size, and the choice of molecular interaction make the calculation of complex, collective and concentration dependent properties extremely difficult. 65 In contrast, a key difficulty in building continuum type models is that they almost always require parameters adjusted to reproduce experiment. The obvious danger of this is that the model becomes an exercise in adjusting fitting parameters, and so will not be predictive and will not reproduce the correct underlying physical mechanisms.

A solution to this problem is to connect the frameworks of molecular simulation with continuum based methods. The strength of continuum based methods is that they allow for the calculation of collective properties over a range of concentrations but must rely on some form of molecularbased input to yield quantitative predictions of collective properties of electrolytes. Essentially this amounts to using AIMD to determine the parameters of the CSM model, so that rather than adjusting parameters to reproduce 
complex experimental collective properties of electrolyte solution, the precise value of the parameters can be determined more rigorously by comparison with the direct intrinsic properties they are designed to match. Some examples are the peak positions in radial distribution functions and condensed phase polarizabilities, which are important to the model of Ref 33 and can be determined from simulation, where they are not available experimentally.

In what follows, we provide new concrete examples of this proposed approach.

\section{Theory}

The key quantity of interest is $W(r)$, the free energy of two ions in water at infinite dilution as a function of separation. This is referred to as the PMF and is related to the radial distribution function by the following expression:

$$
\beta W(r)=-\ln [g(r)]
$$

where $\beta=\left(k_{\mathrm{B}} T\right)^{-1}, g(r)$ is the radial distribution function for the ion pair for a simulation at infinite dilution. We calculate the PMF for $\mathrm{NaCl}$ using this method, the calculation details are given in Ref. 66. These simulations were performed at an effective concentration of $2.5 \mathrm{M}$ as there were $5 \mathrm{NaCl}$ pairs of ions in the box. To confirm that it is reasonable to use this PMF in the infinite dilution limit the PMF was also calculated using Umbrella Sampling(US) with only one cation-anion pair in the simulation box. The resulting PMFs are shown in Figure 1. which shows that they are within error of each other. Although some concentration dependence may be expected due to the change in the Debye-Length, the box size is likely too small to see this effect.

At infinite dilution, the PMF can be divided into two contributions:[58] A short-range term and a long-range Coulomb repulsion:

$$
W(r)=W^{\mathrm{sr}}(r)+\frac{z_{i} z_{j} e^{2}}{4 \pi \epsilon_{o} \epsilon_{w} r}
$$

The small box sizes required to perform AIMD simulations means that the PMF can only be calculated out to a limited separation with any reliability. This is because once the separation becomes bigger than half the box size an ion will be closer to the periodic image of the counter ion then it is to the actual ion. This means the long-range tail of the PMF can not be calculated accurately and there is therefore an arbitrary offset in the energy, as the 0 at infinite separation is not known.

To determine the PMF for larger separations with AIMD we therefore assume that $W^{\mathrm{sr}}(r>7 \AA)=0$, so that the sodium chloride PMF is just given by the Coulomb attraction for separations larger than $7 \AA$. Comparison with classical PMFs show that this is a reasonable assumption. 58. In other words, we adjust the height of the AIMD PMF so that the values with $r>7 \AA$ are as close as possible to

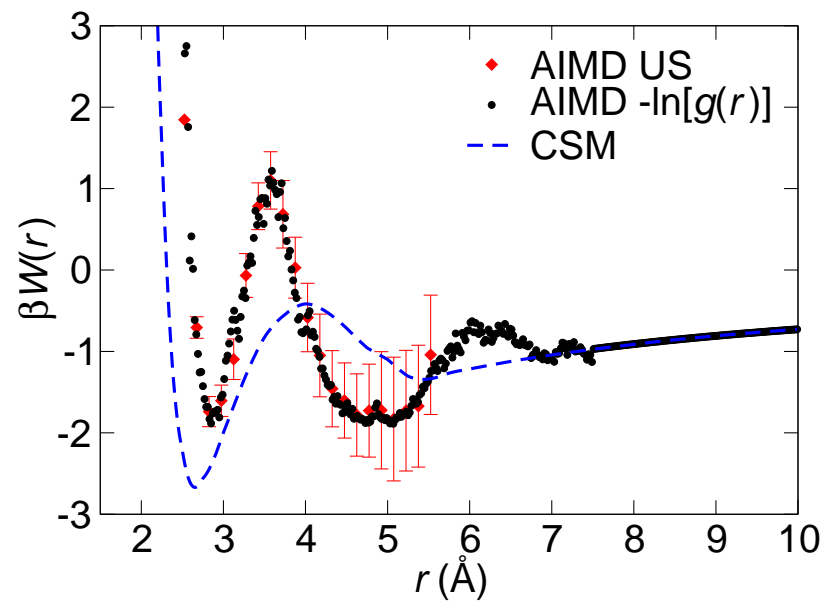

Figure 1: Comparison of sodium chloride PMF calculated with AIMD using $-\ln [g(r)]$ (black) and umbrella sampling(red) and the CSM(blue). The long-range Coulomb attraction is used for $r>7.5 \AA$ for the AIMD PMF.

the Coulomb interaction. The potentials determined from AIMD simulation in this way are shown in Figure 1.

Ref. 63 uses Car Parinello MD with BLYP with no dispersion correction to calculate the $\mathrm{NaCl} \mathrm{PMF}$. The resulting potential is very similar to the one calculated here. This is gratifying and contrasts strongly with the dramatic model dependence observed for classical water models. The degree of agreement may be somewhat fortuitous however as it likely relies on a fortuitous cancellation of ion-ion and ion-water dispersion interactions. This agreement may not be so good for other ions therefore. 30.

We aim to correct the over-attraction of cation-anion pairs with the CSM of Ref. 53 by comparing with the AIMD based PMF. To do so we begin with the expression for the infinite dilution interaction potential of a cationanion pair as given by Eq. 1 in Ref. 53 .

$$
W(r)=W_{\mathrm{COSMO}}(r)+\Delta W_{\mathrm{cav}}(r)+\Delta W_{\mathrm{disp}}(r)
$$

These three terms are described in Ref. 53 where they are labelled as $G$ instead of $W$ as they correspond to Gibbs free energies. Essentially this expression amounts to two things: an ab initio calculation of the direct ion-ion interaction at the MP2 level, where the two ions are placed inside cavities in a dielectric medium. This is calculated using the Conductor Like Screening Model (COSMO). Crucially the overlap of the dielectric interface changes shape as the two ions overlap. The second contribution, which makes up the second two terms is simply a typical solvent accessible surface area calculation, but the parameters for the surface tension parameter is specific to each ion and based on an accurate treatment of the dispersion interaction, and is consistent with the solvation energy of the ions. 
In this paper, these are calculated with the size parameters of the ions adjusted to reproduce the single ion solvation energies. The resulting size parameters are very close to experimental estimates of the same quantities. [53] The long-range Coulomb attraction is included in the $W_{\mathrm{COSMO}}(r)$ term.

It is likely that this over attraction arises from an overestimation of the Coulomb attraction, at small separations when the solvent is not present between the ions. This suggests that a Coulomb repulsion which is proportional to the solvent accessible surface area will be a reasonable correction, this needs to be combined with a damping of the potential to reduce them to a reasonable size. We suggest the following mathematical form to replace Eq3

$$
\begin{aligned}
W(r)= & \frac{1}{\lambda}\left(W_{\mathrm{COSMO}}(r)-W^{\mathrm{lr}}(r)+\Delta W_{\text {cav }}(r)+\Delta W_{\mathrm{disp}}(r)\right. \\
& \left.+\frac{\eta}{r} \frac{\Delta A_{i}(r)+\Delta A_{j}(r)}{A_{i}+A_{j}}\right)+W^{\mathrm{lr}}(r)
\end{aligned}
$$

Where $\lambda$ and $\eta$ are fitted parameters. $A_{i}$ and $A_{j}$ give the total solvent accessible surface area of the two ions, and $\Delta A_{i}(r)$ and $\Delta A_{j}(r)$ give the change in that area as the two ions come together. Again, see Ref. 53 for details. The term in the brackets is obviously equivalent to $W^{\mathrm{sr}}(r)$.

To determine the value for the fitted parameters we can use the AIMD PMF. The total overall strength of the ion-ion interaction can be estimated using the expression for the second virial coefficient:

$$
B=-2 \pi \int_{0}^{7} d r r^{2}\left(e^{-\beta W(r)}-1\right)
$$

where 7 is where both potentials have converged to the Coulomb potential. This value can be estimated for $\mathrm{NaCl}$ with the AIMD PMF, the $\lambda$ and $\eta$ parameters used in Eq. 4 can then be adjusted so that the CSM PMF for $\mathrm{NaCl}$ also has the same value for the $B$ coefficient and therefore has the same overall attraction. Agreement with experimental osmotic coefficients was also taken into account in choosing the values for these parameters. The resulting values are: $\lambda=3$ and $\eta=120$, which gives $B=565 \AA$ for the $\mathrm{NaCl}$ potential calculated with AIMD and with the CSM.

A comparison of the AIMD and CSM PMF for $\mathrm{NaCl}$ is shown in Figure 1. It is clear that the repulsive barrier between the CIP and SSIP with the CSM is too wide by comparison with the $a b$ initio PMF. Obviously the depth of the contact well is not reproduced with the CSM, this is because it does not have a SSIP well and so the contact well needs to account for this effectively. This means that with these potentials, it will not be possible to accurately treat dynamical properties of ions in solution, which is not surprising.

However, we can use Eq. 4 to calculate PMFs for all 20 of the alkali-halide salts. Using the same values for the $\lambda$ and $\eta$ parameters. The resulting PMFs are shown in Figure 2.

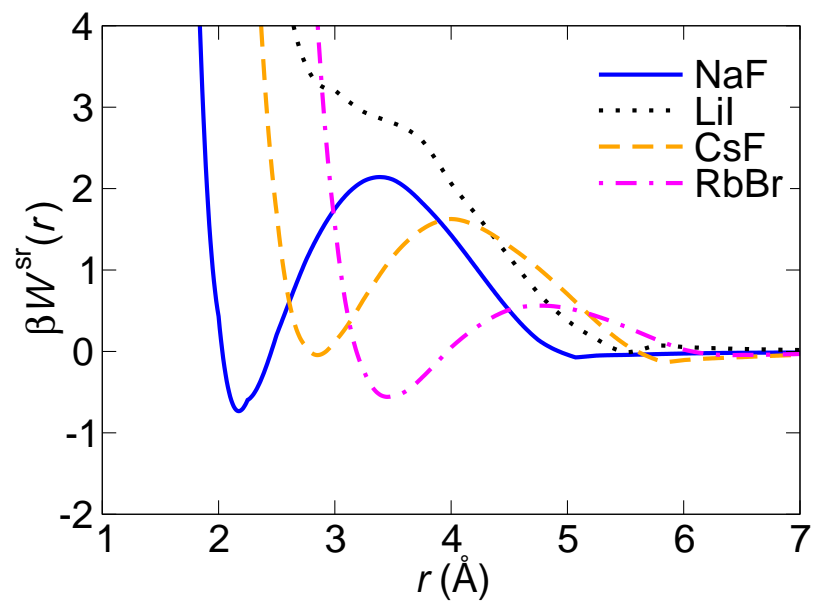

Figure 2: Some of the short-range PMFs calculated with the CSM. The Coulomb attraction has been removed.

Ref 53 makes some general conclusions about these potentials that still stand and are worth restating here. Firstly, these potentials follow the like-prefers-like effect, where ions of similar size are more strongly attracted to each other. This property is also exhibited in the experimental osmotic coefficients. The second conclusion for two ions to come into contact they must remove the water from between them, and the free energy cost associated with this process is an important part in determining the PMF of two ions interacting.

The third conclusion is that dispersion plays a significant role in these interactions and can not be neglected. Firstly, dispersion is necessary to get water structure right as evidenced by the improvement in the density when it is included in DFT simulations of water. Secondly ionwater dispersion is an important part of the total solvation energy of ions and therefore getting it correct is necessary to determine the free energy cost of desolvating the ion. Thirdly direct ion-ion dispersion interaction is important for large cation-anion interactions, where it plays a role in stabilizing the large-large ion attraction discussed earlier. 67.

Simple DFT functionals do not treat dispersion interactions accurately and so improving their description is important for an accurate AIMD description of ions in solution.

\section{Calculation Details}

Umbrella sampling is used to obtain the ion-pairing free-energy of $\mathrm{Na}^{+}$and $\mathrm{Cl}^{-}$under bulk periodic boundary conditions. The system contains a single $\mathrm{Na}^{+}$and $\mathrm{Cl}^{-}$ and 110 water molecules in a $15.198 \times 15.198 \times 15.198 \AA^{3}$ supercell. Sampling windows for the $\mathrm{Na}^{2+} \ldots \mathrm{Cl}^{-}$distance 
ranging from 2.2 to $5.6 \AA$ were equally spaced by $0.2 \AA$ employing harmonic umbrella potentials of the from $V(r)=$ $k\left(r_{0}-r\right)^{2}$ with a force constant $k$ of $40.0 \mathrm{~kJ} \mathrm{~mol}^{-1} \AA^{-2}$. For each window a NVT (at $300 \mathrm{~K}$ ) simulation is performed under periodic boundary conditions using CP2K simulation suite (http:www.cp2k.org) with the QuickStep module for the DFT calculations. 68] The protocol given in Ref. 21, using a double zeta basis set that has been optimized for the condensed phase 69 in conjunction with GTH pseudopotentials [70] using a 400 Ry cutoff for the auxiliary plane wave basis is followed. A Nosé-Hoover thermostat was attached to every degree of freedom to ensure equilibration 71. The Becke exchange 72 and correlation due to Lee, Yang and Parr (LYP) [73] is utilized in addition to the dispersion correction (D2) put forth by Grimme 74 with a $40 \AA$ cut-off. For each umbrella window, a trajectory of at least 50 ps was collected after 5 ps of equilibration and the free-energy profiles was extracted using the weighted histogram analysis method. 75]

\section{Experimental Comparison}

To test the resulting PMFS we can follow Ref. 76 , which utilizes the Hyper-netted Chain (HNC) closure of the Ornstein-Zernike (OZ) equation to calculate the osmotic coefficients of electrolyte solutions at finite concentration using the infinite dilution PMF. To do this we use the pyOZ program 77 ] developed by Luboš Vrbka, an iterative OZ equation solver. Following Ref. 78 and 58 , we use a concentration dependent form of the dielectric function of water to account approximately for many-body effects that will become more important as the ion concentration increases:

$$
\epsilon(c)=\frac{78.3}{1+0.19 c}
$$

where $c$ is the concentration of salt in Molarity, and 0.19 is a constant determined by fitting to experimental data for six different salts and averaging. 58 Ref. 58 shows that the osmotic coefficients at the concentrations we are interested in are dominated by the cation-anion interaction potential. We therefore use a simple hard-sphere repulsion with a Coulomb repulsion for this interaction, as using a more realistic form for this potential does not alter the results significantly.

We can therefore use the infinite dilution PMF calculated with both the CSM and with AIMD, and compare to experimental values. This provides an important test of the model as osmotic coefficients are a central example of a collective property and the ability to reproduce them displays the power of these approaches to treating electrolyte solutions. The results of this calculation are presented in Figure 3, 4 and 5 .

The experimental osmotic coefficients are calculated with the Pitzer equations [7] after adjustment from the Lewis-Randal to McMillan-Mayer systems. 80, 81.

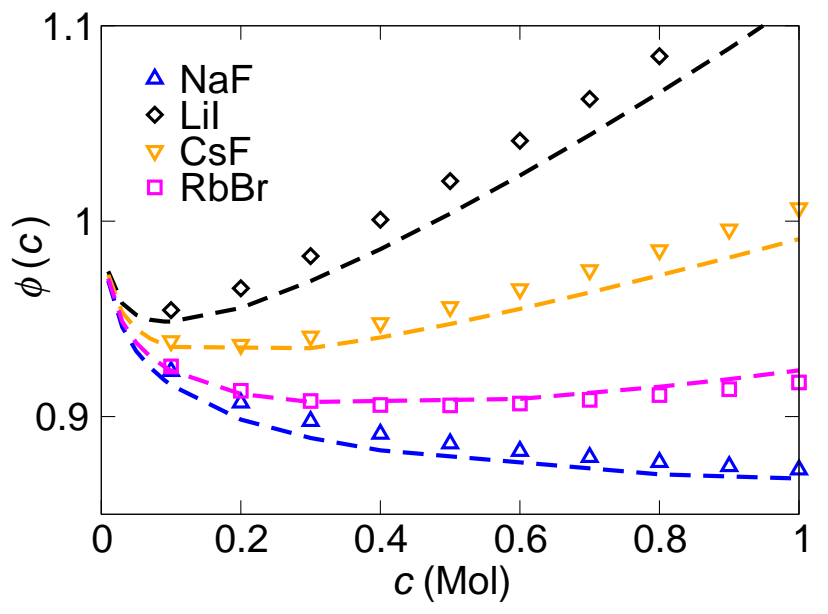

Figure 3: Comparison of experimental and theoretical osmotic coefficients. The theoretical values (dashed lines) are calculated with the CSM. The experimental values (symbols) are determined from Ref. 79] after conversion to the Macmillan Mayer scheme. 80]

We can see that the osmotic coefficients of all the salts are reproduced reasonably well once the ion-ion CSM PMFs are corrected with information from the $a b$ initio calculations. In particular Figure 5 shows that the theoretical values match the experimental ones with $R^{2}=0.94$, a very good correlation considering the simplicity of the model. This is an important and impressive result. The experimental agreement of these results exceeds that of any CMD approach, which would require much more computational time. Reproducing these properties has long been an elusive goal of the physical chemistry community and represents a significant step forward in our understanding of these properties. This model represents the achievement of a goal outlined in this same journal 5 years ago in Ref. 82, where the ambition was to model the direct ionion interaction accurately enough in solution so that one or two globally fitted parameters could capture the effects of hydration that a CSM necessarily neglects.

This amounts indirectly to a model of activities of salt solutions as well because osmotic coefficients can be converted to activities via standard thermodynamic relations. We can only reasonably hope this model will be accurate up to 1 or $2 \mathrm{M}$ because changes in the two body interaction will occur at higher concentrations due to changes in the water structure. 2] Calculation of osmotic coefficients at higher density will likely require alternative methods such as Ref. 83 .

The only other models that we are aware of in the literature, which do not rely on parameters adjusted for each salt are Ref. 84 and Ref. 85. However, a crucial omission is that these approaches did not treat fluoride, as without the fluoride salts the reversal in ordering of the ion pairs is barely discernible and so it is much easier to build models that reproduce experiment without salt specific fitted 


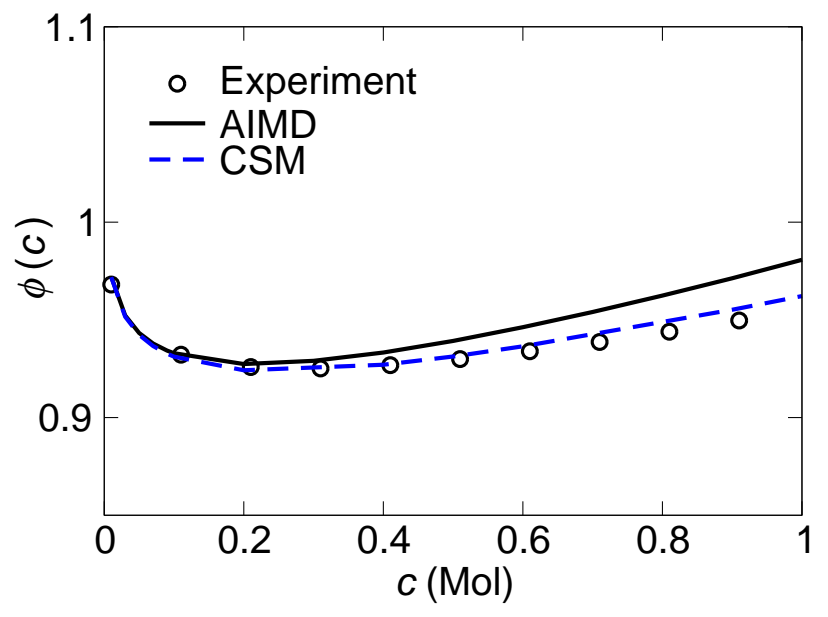

Figure 4: Osmotic Coefficients for $\mathrm{NaCl}$ calculated with AIMD and CSM compared with experiment.

parameters. Also, these models can not explain intrinsic properties of an ion such as solvation energies or properties of an ion near an interface such as surface tensions, whereas this model can.

Future work will involve generalizations to non-aqueous solutions, [86, 87] to more complex ions, i.e., divalent and organic, 21, 30 and to more complex molecules such as charged carboxylates and to a wider range of properties such as solvation energies and surface tensions. This will hopefully allow us to build useful, predictive and quantitatively accurate models of complex Hofmeister effects.

\section{Conclusions}

We have advocated for an approach to modeling ionion interactions that incorporates information from stateof-the-art AIMD simulation into reduced models of ions in solution. These reduced models can then be used to calculate collective and concentration dependent properties of electrolyte solution that would be too difficult to do with AIMD directly. We showed an example of this approach, where the short-range AIMD PMF of $\mathrm{NaCl}$ was combined with a long-range Coulomb attraction, which was then used in the OZ equation with HNC closure to accurately calculate the osmotic pressure of Sodium Chloride. The AIMD PMF for $\mathrm{NaCl}$ was then used to improve and parametrize a previously developed CSM of ion-ion interactions. This allowed the osmotic pressure for all 20 of the alkali-halide salts to be calculated accurately up to $1 \mathrm{M}$. This approach of connecting frameworks is essential to provide descriptions of electrolyte solutions that are both quantitatively predictive and practically useful.

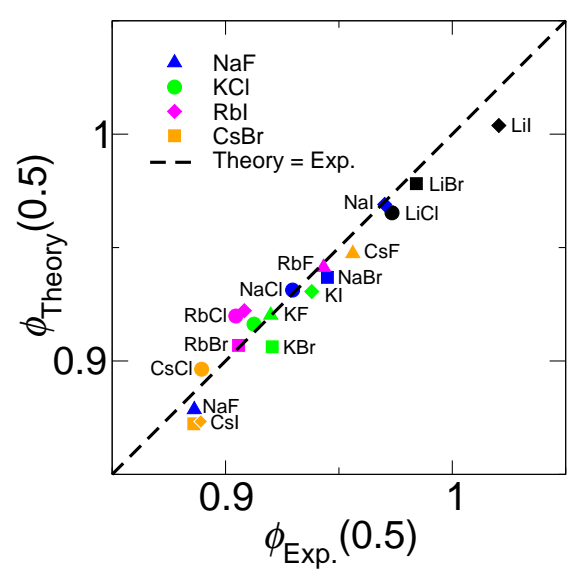

Figure 5: Osmotic Coefficients calculated at 0.5 M for 19 alkali halide salts compared with experimental values.

\section{Acknowledgement}

This research used resources of the National Energy Research Scientific Computing Center, a DOE Office of Science User Facility supported by the Office of Science of the U.S. Department of Energy under Contract No. DE-AC02-05CH11231. TD and CJM were supported by the U.S. Department of Energy, Office of Science, Office of Basic Energy Sciences, Division of Chemical Sciences, Geosciences, and Biosciences. MDB was supported by $\mathrm{MS}^{3}$ (Materials Synthesis and Simulation Across Scales) Initiative, a Laboratory Directed Research and Development Program at Pacific Northwest National Laboratory (PNNL). PNNL is a multiprogram national laboratory operated by Battelle for the U.S. Department of Energy.

\section{References}

[1] D. E. Otten, P. R. Shaffer, P. L. Geissler, R. J. Saykally, Elucidating the Mechanism of Selective Ion Adsorption to the Liquid Water Surface., Proc. Natl. Acad. Sci. U. S. A. 109 (2012) 31903190 .

[2] Z. Cao, J. F. Dama, L. Lu, G. A. Voth, Solvent Free Ionic Solution Models from Multiscale Coarse-Graining, J. Chem. Theory Comput. 9 (2013) 172-178.

[3] T. T. Duignan, D. F. Parsons, B. W. Ninham, Ion Interactions with the Air-Water Interface using a Continuum Solvent Model, J. Phys. Chem. B 118 (2014) 8700-8710.

[4] N. Schwierz, D. Horinek, R. R. Netz, Anionic and Cationic Hofmeister Effects on Hydrophobic and Hydrophilic Surfaces, Langmuir 29 (2013) 2602-2614.

[5] Y. Levin, A. P. dos Santos, Ions at Hydrophobic Interfaces., J. Phys.: Condens. Matter 26 (2014) 203101.

[6] R. Wang, Z.-G. Wang, Continuous Self-Energy of Ions at the Dielectric Interface, Phys. Rev. Lett. 112 (2014) 136101.

[7] A. P. Lyubartsev, A. Laaksonen, Osmotic and Activity Coefficients from Effective Potentials for Hydrated Ions, Phys. Rev. E 55 (1997) 5689-5696**(Very early CMD calculation of osmotic coefficients).

[8] V.-T. Pham, J. L. Fulton, Ion-Pairing in Aqueous $\mathrm{CaCl}_{2}$ and RbBr Solutions: Simultaneous Structural Refinement of XAFS 
and XRD Data., J. Chem. Phys. 138 (2013) 044201**(Presents a new approach for co-refinement of XRD and EXAFS to provide highly accurate experimental description of structure surrounding ion pairs).

[9] M. Galib, G. Hanna, Mechanistic Insights into the Dissociation and Decomposition of Carbonic Acid in Water via the Hydroxide Route: An Ab Initio Metadynamics Study., J. Phys. Chem. B 115 (2011) 15024-15035.

[10] R. Hartkamp, B. Coasne, Structure and Transport of Aqueous Electrolytes: From Simple Halides to Radionuclide Ions., J. Chem. Phys. 141 (2014) 124508.

11] R. Semiat, Critical Review Energy Issues in Desalination Processes, Environmental Science \& Technology 42 (2008) 81938201 .

[12] S. Shimizu, W. M. McLaren, N. Matubayasi, The Hofmeister Series and Protein-Salt Interactions., J. Chem. Phys. 124 (2006) 234905.

[13] Y. Zhang, P. S. Cremer, Interactions Between Macromolecules and Ions: The Hofmeister Series., Curr. Opin. Chem. Biol. 10 (2006) 658-663.

[14] M. Lund, L. Vrbka, P. Jungwirth, Specific Ion Binding to Nonpolar Surface Patches of Proteins., J. Am. Chem. Soc. 130 (2008) 11582-11583.

[15] D. F. Parsons, B. W. Ninham, Surface Charge Reversal and Hydration Forces Explained by Ionic Dispersion Forces and Surface Hydration, Colloids Surf., A 383 (2011) 2-9.

[16] A. Salis, F. Cugia, D. F. Parsons, B. W. Ninham, M. Monduzzi, Hofmeister Series Reversal for Lysozyme by Change in $\mathrm{pH}$ and Salt Concentration: Insights from Electrophoretic Mobility Measurements, Phys. Chem. Chem. Phys. 14 (2012) 4343-4346.

[17] P. Jungwirth, P. S. Cremer, Beyond Hofmeister, Nat. Chem. 6 (2014) 261-263.

[18] N. Schwierz, D. Horinek, R. R. Netz, Specific Ion Binding to Amphiprotic Surface Groups and the $\mathrm{pH}$ Dependence of the Hofmeister Series, Langmuir 31 (2015) 215-225.

[19] J. De Yoreo, P. Gilbert, N. Sommerdijk, R. Penn, S. Whitelam, D. Joester, H. Zhang, J. Rimer, A. Navrotsky, J. Banfield, A. Wallace, F. Michel, F. Meldrum, H. Cölfen, P. Dove, Crystallization by Particle Attachment in Synthetic, Biogenic, and Geologic Environments, Science 349 (2015) aaa6760.

[20] E. Gallicchio, K. Paris, R. M. Levy, The AGBNP2 Implicit Solvation Model., J. Chem. Theory Comput. 5 (2009) 25442564

[21] E. Pluhařová, M. D. Baer, C. J. Mundy, B. Schmidt, P. Jungwirth, Aqueous Cation-Amide Binding: Free Energies and IR Spectral Signatures by Ab Initio Molecular Dynamics, J. Phys. Chem. Lett. 5 (2014) 2235-2240.

[22] V. Venkateshwaran, S. Vembanur, S. Garde, Water-Mediated Ion-Ion Interactions are Enhanced at the Water Vapor-Liquid Interface, Proc. Natl. Acad. Sci. U. S. A. 111 (2014) 8729-8734.

[23] A. P. dos Santos, Y. Levin, Electrolytes Between Dielectric Charged Surfaces: Simulations and Theory, J. Chem. Phys. 142 (2015) 194104.

[24] J. L. Fulton, G. K. Schenter, M. D. Baer, C. J. Mundy, L. X. Dang, M. Balasubramanian, Probing the Hydration Structure of Polarizable Halides: A Multiedge XAFS and Molecular Dynamics Study of the Iodide Anion., J. Phys. Chem. B 114 (2010) 12926-129337.

[25] P. Hünenberger, M. Reif, Single-Ion Solvation: Experimental and Theoretical Approaches to Elusive Thermodynamic Quantities, The Royal Society of Chemistry, 2011*(Very useful comprehensive review of the literature on single ion properties in solution).

[26] J. L. Fulton, S. M. Kathmann, G. K. Schenter, M. Balasubramanian, Hydrated Structure of $\mathrm{Ag}(\mathrm{I})$ Ion from SymmetryDependent, K- and L-Edge XAFS Multiple Scattering and Molecular Dynamics Simulations, J. Phys. Chem. A 113 (2009) 13976-13984.

[27] E. Cauët, S. Bogatko, J. H. Weare, J. L. Fulton, G. K. Schenter, E. J. Bylaska, Structure and Dynamics of the Hydration Shells of the $\mathrm{Zn}^{2+}$ Ion from Ab Initio Molecular Dynamics and Com- bined Ab Initio and Classical Molecular Dynamics Simulations., J. Chem. Phys. 132 (2010) 194502.

$28]$ M. D. Baer, V.-T. Pham, J. L. Fulton, G. K. Schenter, M. Balasubramanian, C. J. Mundy, Is Iodate a Strongly Hydrated Cation?, J. Phys. Chem. Lett. 2 (2011) 2650-2654.

[29] S. Bogatko, E. Cauët, E. Bylaska, G. Schenter, J. Fulton, J. Weare, The Aqueous $\mathrm{Ca}^{2+}$ System, in Comparison with $\mathrm{Zn}^{2+}, \mathrm{Fe}^{3+}$, and $\mathrm{Al}^{3+}$ : An Ab Initio Molecular Dynamics Study, Chem. - Eur. J. 19 (2013) 3047-3060.

30] M. D. Baer, C. J. Mundy, Local Aqueous Solvation Structure around $\mathrm{Ca}^{2+}$ during $\mathrm{Ca}^{2+}-\mathrm{Cl}^{-}$Pair Formation, J. Phys. Chem. B (2016) Just-Accepted $* *$ (Provides an accurate description of the structure of these ions both alone and as a pair using AIMD-DFT).

[31] D. Horinek, S. I. Mamatkulov, R. R. Netz, Rational Design of Ion Force Fields Based on Thermodynamic Solvation Properties, J. Chem. Phys. 130 (2009) 124507*(Rigorous examination of the determination of parameters for classical simulation).

32] M. M. Reif, P. H. Hünenberger, Computation of MethodologyIndependent Single-Ion Solvation Properties from Molecular Simulations. IV. Optimized Lennard-Jones Interaction Parameter Sets for the Alkali and Halide Ions in Water., J. Chem. Phys. 134 (2011) 144104.

[33] T. T. Duignan, D. F. Parsons, B. W. Ninham, A Continuum Model of Solvation Energies Including Electrostatic, Dispersion, and Cavity Contributions., J. Phys. Chem. B 117 (2013) 94219429

[34] V. S. Bryantsev, M. S. Diallo, W. A. Goddard, Calculation of Solvation Free Energies of Charged Solutes Using Mixed Cluster/Continuum Models, J. Phys. Chem. B 112 (2008) 97099719

[35] T. L. Beck, The Influence of Water Interfacial Potentials on Ion Hydration in Bulk Water and Near Interfaces, Chem. Phys. Lett. 561-562 (2013) 1-13.

[36] D. Asthagiri, L. R. Pratt, M. E. Paulaitis, S. B. Rempe, Hydration Structure and Free Energy of Biomolecularly Specific Aqueous Dications, Including $\mathrm{Zn}^{2+}$ and First Transition Row Metals, J. Am. Chem. Soc. 126 (2004) 1285-1289.

[37] D. Asthagiri, L. R. Pratt, J. D. Kress, Ab Initio Molecular Dynamics and Quasichemical Study of $\mathrm{H}^{+}(\mathrm{aq})$., Proc. Natl. Acad. Sci. U. S. A. 102 (2005) 6704-6708.

[38] Y. Marcus, Ion Properties, CRC Press, 1997.

[39] R. Schmid, A. M. Miaha, V. N. Sapunovb, A New Table of the Thermodynamic Quantities of Ionic Hydration: Values and Some Applications (Enthalpy-Entropy Compensation and Born Radii), Phys. Chem. Chem. Phys. 2 (2000) 97-102.

[40] R. C. Remsing, M. D. Baer, G. K. Schenter, C. J. Mundy, J. D. Weeks, The Role of Broken Symmetry in Solvation of a Spherical Cavity in Classical and Quantum Water Models, J. Phys. Chem. Lett. 5 (2014) 2767-2774.

41] K. Leung, S. B. Rempe, O. A. von Lilienfeld, Ab Initio Molecular Dynamics Calculations of Ion Hydration Free Energies., J. Chem. Phys. 130 (2009) 204507*(Only example of AIMD determination of ionic solvation free energies.)

[42] H.-S. Lee, M. E. Tuckerman, Ab Initio Molecular Dynamics Studies of the Liquid-Vapor Interface of an $\mathrm{HCl}$ Solution., J. Phys. Chem. A 113 (2009) 2144-2151.

[43] M. D. Baer, I.-F. W. Kuo, H. Bluhm, S. Ghosal, Interfacial Behavior of Perchlorate Versus Chloride Ions in Aqueous Solutions., J. Phys. Chem. B 113 (2009) 15843-15850.

[44] C. J. Mundy, I. F. W. Kuo, M. E. Tuckerman, H. S. Lee, D. J. Tobias, Hydroxide Anion at the Air-Water Interface, Chem. Phys. Lett. 481 (2009) 2-8.

[45] M. D. Baer, C. J. Mundy, Toward an Understanding of the Specific Ion Effect Using Density Functional Theory, J. Phys. Chem. Lett. 2 (2011) 1088-1093.

[46] M. D. Baer, I.-F. W. Kuo, D. J. Tobias, C. J. Mundy, Toward a Unified Picture of the Water Self-Ions at the Air-Water Interface: A Density Functional Theory Perspective., J. Phys. Chem. B 118 (2014) 8364-8372.

[47] Y. Levin, A. P. dos Santos, A. Diehl, Ions at the Air-Water 
Interface: An End to a Hundred-Year-Old Mystery?, Phys. Rev. Lett. 103 (2009) 257802.

[48] T. T. Duignan, D. F. Parsons, B. W. Ninham, Hydronium and Hydroxide at the Air-Water Interface with a Continuum Solvent Model, Chem. Phys. Lett. 635 (2015) 1-12.

[49] R. R. Netz, D. Horinek, Progress in Modeling of Ion Effects at the Vapor/Water Interface., Annu. Rev. Phys. Chem. 63 (2012) 401-418.

[50] R. A. Robinson, R. H. Stokes, Electrolyte Solutions, Butterworth \& Co., 1959

[51] H. L. Friedman, C. V. Krishnan, Ionic Interactions in Water, Ann. N. Y. Acad. Sci. 204 (1973) 78-99.

[52] W. Kunz, L. Belloni, O. Bernard, B. W. Ninham, Osmotic Coefficients and Surface Tensions of Aqueous Electrolyte Solutions: Role of Dispersion Forces, J. Phys. Chem. B 108 (2004) 23982404

[53] T. T. Duignan, D. F. Parsons, B. W. Ninham, A Continuum Solvent Model of Ion-Ion Interactions in Water., Phys. Chem. Chem. Phys. 16 (2014) 22014-22027**(Reproduces the ion specificity of osmotic coefficients with a simple model with low computational demand).

[54] T. T. Duignan, D. F. Parsons, B. W. Ninham, A Continuum Solvent Model of the Partial Molar Volumes and Entropies of Ionic Solvation, J. Phys. Chem. B 118 (2014) 3122-3132.

[55] M. Kohagen, P. E. Mason, P. Jungwirth, Accurate Description of Calcium Solvation in Concentrated Aqueous Solutions, J. Phys. Chem. B 118 (2014) 7902-7909.

[56] M. Kohagen, P. E. Mason, P. Jungwirth, Accounting for Electronic Polarization Effects in Aqueous Sodium Chloride via Molecular Dynamics Aided by Neutron Scattering, J. Phys. Chem. B (2015) ASAP**(Presents a means of correcting nonpolarizable simulations and shows good agreement with neutron scattering experiments)

[57] D. E. Smith, L. X. Dang, Computer Simulations of $\mathrm{NaCl}$ Association in Polarizable Water, J. Chem. Phys. 100 (1994) 37573766 .

[58] I. Kalcher, J. Dzubiella, Structure-Thermodynamics Relation of Electrolyte Solutions., J. Chem. Phys. 130 (2009) 134507.

[59] C. J. Fennell, A. Bizjak, V. Vlachy, K. A. Dill, Ion Pairing in Molecular Simulations of Aqueous Alkali Halide Solutions., J. Phys. Chem. B 113 (2009) 6782-6791.

60] M. Fyta, I. Kalcher, J. Dzubiella, L. Vrbka, R. R. Netz, Ionic Force Field Optimization Based on Single-Ion and Ion-Pair Solvation Properties., J. Chem. Phys. 132 (2010) 024911**(Shows parameters need to be adjusted for individual salts to match experimental ion specific trends).

[61] Y. Luo, W. Jiang, H. Yu, A. D. MacKerell, B. Roux, Simulation Study of Ion Pairing in Concentrated Aqueous Salt Solutions with a Polarizable Force Field, Faraday Discuss. 160 (2013) 135149.

[62] A. Saxena, A. E. García, Multisite Ion Model in Concentrated Solutions of Divalent Cations $\left(\mathrm{MgCl}_{2}\right.$ and $\left.\mathrm{CaCl}_{2}\right)$ : Osmotic Pressure Calculations, J. Phys. Chem. B 119 (2015) 219-227.

[63] J. Timko, D. Bucher, S. Kuyucak, Dissociation of $\mathrm{NaCl}$ in Water from Ab Initio Molecular Dynamics Simulations, J. Chem. Phys. 132 (2010) 114510*(Early AIMD calculation of ion-ion interactions).

64] E. Pluhařová, O. Marsalek, B. Schmidt, P. Jungwirth, Ab Initio Molecular Dynamics Approach to a Quantitative Description of Ion Pairing in Water, J. Phys. Chem. Lett. 4 (2013) 4177$4181 *$ (AIMD description of $\mathrm{LiF}$ in water).

[65] Z. Mester, A. Z. Panagiotopoulos, Mean Ionic Activity Coefficients in Aqueous $\mathrm{NaCl}$ Solutions from Molecular Dynamics Simulations, J. Chem. Phys. 142 (2015) 044507.

[66] M. D. Baer, J. L. Fulton, M. Balasubramanian, G. K. Schenter, C. J. Mundy, Persistent Ion Pairing in Aqueous Hydrochloric Acid., J. Phys. Chem. B 118 (2014) 7211-7220.

[67] T. T. Duignan, D. F. Parsons, B. W. Ninham, Collins's Rule, Hofmeister Effects and Ionic Dispersion Interactions, Chem. Phys. Lett. 608 (2014) 55-59.

[68] J. VandeVondele, M. Krack, F. Mohamed, M. Parrinello,
T. Chassaing, J. Hutter, QUICKSTEP: Fast and Accurate Density Functional Calculations using a Mixed Gaussian and Plane Waves Approach, Comp. Phys. Comm. 167 (2005) 103-128.

[69] J. VandeVondele, J. Hutter, Gaussian Basis Sets for Accurate Calculations on Molecular Systems in Gas and Condensed Phases, J. Chem. Phys. 127 (2007) 114105.

[70] S. Goedecker, M. Teter, J. Hutter, Separable Dual-Space Gaussian Pseudopotentials, Phys. Rev. B 54 (1996) 1703-1710.

[71] G. J. Martyna, M. L. Klein, M. E. Tuckerman, Nose-Hoover Chains - The Canonical Ensemble via Continous Dynamics, J. Chem. Phys. 97 (1992) 2635-2643.

[72] A. D. Becke, Density-Functional Exchange-Energy Approximation with Correct Asymptotic Behavior, Phys. Rev. A 38 (1988) 3098-3100.

[73] C. Lee, W. Yang, R. G. Parr, Development of the Colle-Salvetti Correlation-Energy Formula into a Functional of the Electron Density, Phys. Rev. B 37 (1988) 785-789.

[74] S. Grimme, Accurate Description of Van der Waals Complexes by Density Functional Theory Including Empirical Corrections, J. Comput. Chem. 25 (2004) 1463-1473.

[75] J. S. Hub, B. L. De Groot, D. Van Der Spoel, g_wham-A Free Weighted Histogram Analysis implementation Including Robust Error and Autocorrelation Estimates, J. Chem. Theory Comput. 6 (2010) 3713-3720.

[76] L. Vrbka, M. Lund, I. Kalcher, J. Dzubiella, R. R. Netz, W. Kunz, Ion-Specific Thermodynamics of Multicomponent Electrolytes: A Hybrid HNC/MD Approach., J. Chem. Phys. 131 (2009) 154109**(Shows how CMD results can be combined with HNC calculations to predict osmotic coefficients accurately).

[77] L. Vrbka, http://pyoz.vrbka.net (2008).

[78] B. Hess, C. Holm, N. van der Vegt, Modeling Multibody Effects in Ionic Solutions with a Concentration Dependent Dielectric Permittivity, Phys. Rev. Lett. 96 (2006) 147801.

[79] K. S. Pitzer, G. Mayorga, Thermodynamics of Electrolytes. II. Activity and Osmotic Coefficients for Strong Electrolytes with One or Both Ions Univalent, J. Phys. Chem. 77 (1973) 23002308.

[80] H. L. Friedman, Lewis-Randall to McMillan-Mayer Conversion for the Thermodynamic Excess Functions of Solutions. Part II. Excess Energy and Volume, J. Solution Chem. 1 (1972) 413417.

[81] J.-P. Simonin, Study of Experimental-to-McMillan-Mayer Conversion of Thermodynamic Excess Functions, J. Chem. Soc., Faraday Trans. 92 (1996) 3519-3523.

[82] B. W. Ninham, T. T. Duignan, D. F. Parsons, Approaches to Hydration, Old and New: Insights through Hofmeister Effects, Curr. Opin. Colloid Interface Sci. 16 (2011) 612-617.

[83] Y. Luo, B. Roux, Simulation of Osmotic Pressure in Concentrated Aqueous Salt Solutions, J. Phys. Chem. Lett. 1 (2010) 183-189.

[84] J. J. Molina, J.-F. Dufrêche, M. Salanne, O. Bernard, P. Turq, Primitive Models of Ions in Solution from Molecular Descriptions: A Perturbation Approach, J. Chem. Phys. 135 (2011) 234509.

[85] Y. V. Kalyuzhnyi, V. Vlachy, K. A. Dill, Aqueous Alkali Halide Solutions: Can Osmotic Coefficients be Explained on the Basis of the Ionic Sizes Alone?, Phys. Chem. Chem. Phys. 12 (2010) 6260-6266

[86] N. Peruzzi, B. W. Ninham, P. Lo Nostro, P. Baglioni, Hofmeister Phenomena in Nonaqueous Media: The Solubility of Electrolytes in Ethylene Carbonate., J. Phys. Chem. B 116 (2012) 14398-14405.

[87] A. Arslanargin, A. Powers, T. L. Beck, S. W. Rick, Models of Ion Solvation Thermodynamics in Ethylene Carbonate and Propylene Carbonate, J. Phys. Chem. B (2015) 150826112023003. 


\section{Graphical Abstract}

1.

I.

I

I: $1:$

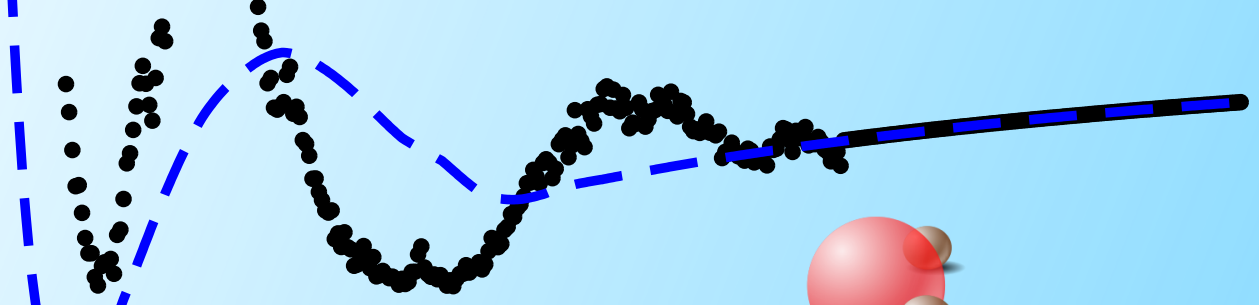

$1 \%$

I/

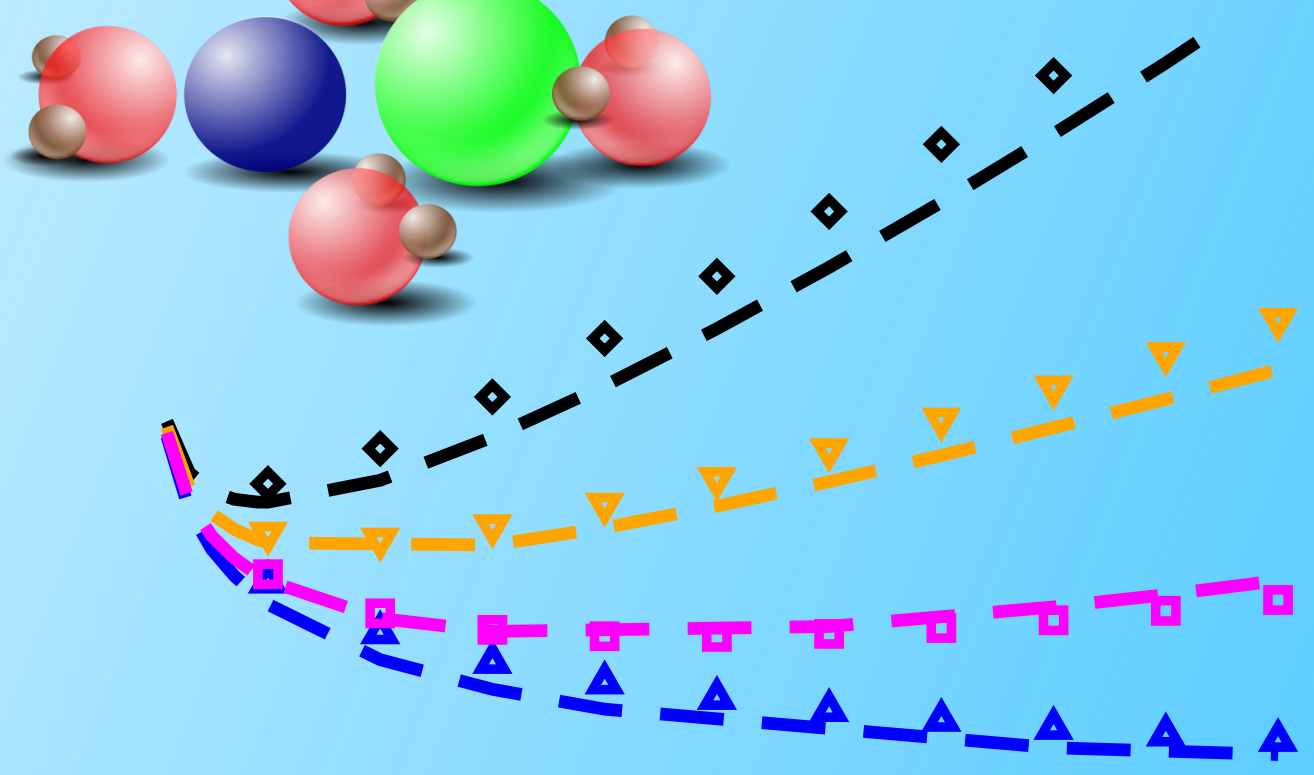

